\title{
Case Report \\ Enterobius vermicularis in the kidney: an unusual location
}

Correspondence
Estelle Cateau
estelle.cateau@chu-poitiers.fr

Received 3 February 2010

Accepted 8 April 2010

\author{
Estelle Cateau, ${ }^{1}$ Mokrane Yacoub, ${ }^{2}$ Christian Tavilien, ${ }^{3}$ \\ Bertrand Becq-Giraudon ${ }^{4}$ and Marie-Hélène Rodier ${ }^{1}$
${ }^{1}$ Laboratoire de Parasitologie et Mycologie Médicale, CHU de Poitiers, 2 rue de la Milétrie, 86021 Poitiers Cedex, France
${ }^{2}$ Laboratoire d'Anatomie et Cytologie Pathologiques, $\mathrm{CHU}$ de Poitiers, 2 rue de la Milétrie, 86021 Poitiers Cedex, France
${ }^{3}$ Service de Chirurgie, Centre Hospitalier, 2 rue Henri Dunant, 86501 Montmorillon, France
${ }^{4}$ Service des Maladies Infectieuses et Tropicales, $\mathrm{CHU}$ de Poitiers, 2 rue de la Milétrie, 86021 Poitiers Cedex, France

\begin{abstract}
A woman was admitted to hospital with abdominal pain. A large kidney stone was recovered and a nephrectomy was performed. Histology revealed the unusual presence of multiple Enterobius vermicularis ova. However, no other parasitic element was recovered on further investigations.
\end{abstract}

\section{Case report}

A 51-year-old woman was admitted to hospital with a left-sided abdominal pain which had been evolving for a few days. She had no remarkable medical history, except a bilateral congenital hip dislocation. Upon examination, she had no fever, and the palpation showed a generalized soft abdomen with left flank and back pain.

The abdominal CT scan found a large kidney stone with calyceal location and calyx dilatation and an aspect of the renal parenchyma consistent with pyonephrosis. The biological investigations at admission showed a white blood cell count of $13400 \mathrm{~mm}^{-3}$ without eosinophilia. The collected urine sample was turbid and routine examination revealed the presence of red blood cells and pus cells $>10000 \mathrm{~mm}^{-3}$. Urine was plated on blood agar and MacConkey agar, and incubated at $37{ }^{\circ} \mathrm{C}$, but no microorganism was recovered. Other laboratory tests were also negative. Given the persistence of symptoms, nephrectomy was decided upon, and the patient was treated with amoxicillin and gentamicin. Histology revealed signs of a chronic kidney inflammation with necrotic areas and granuloma formations. In the periphery of the necrotic area, multiple Enterobius vermicularis ova were recovered (Fig. 1). No adult worms were identified in the examined samples. No worms or eggs were recovered in stools or after the application of a transparent adhesive tape to the perianal area (scotch tape test). The epidemiological investigations and the morphology of the eggs excluded the possibility of other parasitic species. After 4 weeks, the patient rapidly recovered and was not treated for the enterobiasis.

\section{Discussion}

E. vermicularis is one of the most common nematodes in the world. In Western Europe, the prevalence rates in some communities can reach 30-50\% (Burkhart \& Burkhart, 2005), and enterobiasis appears to be more common in temperate than in tropical countries. Following the ingestion of infective eggs, the larvae hatch in the small intestine then the adults settle predominantly in the colon, particularly the caecum, and the terminal ileum. The life cycle of E. vermicularis occurs entirely in the gastrointestinal tract lumen. The gravid females of this roundworm migrate at night through the anus and lay their eggs in the perianal area. The eggs of E. vermicularis, naturally transparent and colourless, measure 50$60 \mu \mathrm{m} \times 20-30 \mu \mathrm{m}$, and are ovoid and asymmetrical, one side being more convex than the other. They embryonate in $6 \mathrm{~h}$ and can remain viable for 20 days in the environment (Burkhart \& Burkhart, 2005). By the resultant scratching of perianal or perineal areas, these eggs are frequently transferred to the fingers, then to the mouth, ensuring autoinfection. Consequently, the diagnosis of enterobiasis can be made by application of a transparent adhesive tape to the perianal area in the morning, before defecation or washing. In some cases, as a consequence of this parasitic migration, some worms find their way to the adjacent orifices, most commonly the female genitourinary tract.

Studies have reported the association between E. vermicularis and the appendix (Aydin, 2007; Cook, 1994; Da Silva et al., 2007; Ramezani \& Dehghani, 2007), but extraintestinal localizations have also been reported, such as the female genital tract (Burkhart \& Burkhart, 2005; Dundas 


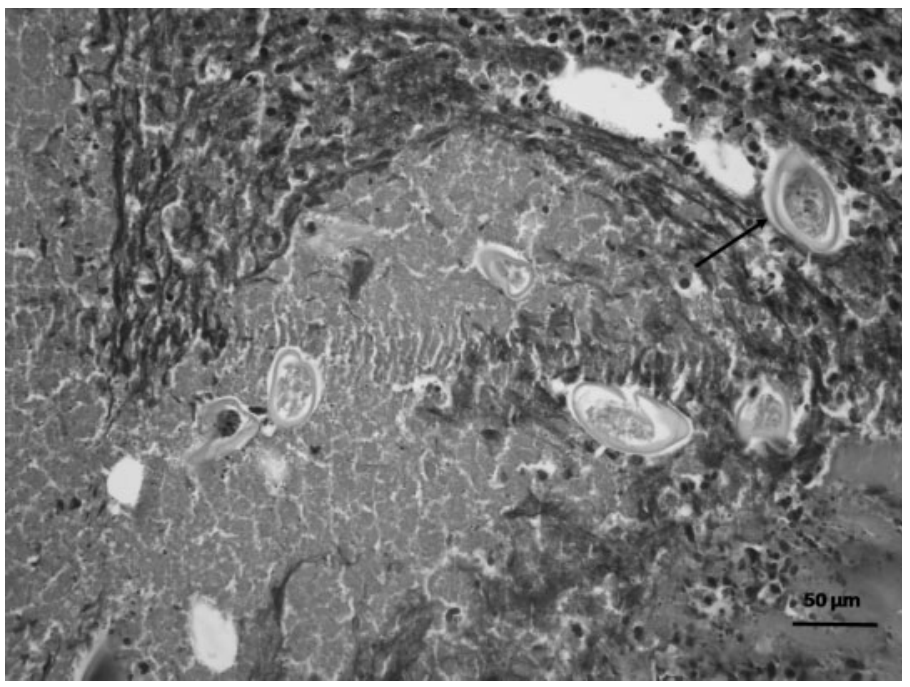

Fig. 1. Ova of Enterobius vermicularis in a kidney sample (HES stain; original magnification $\times 400$ ).

et al., 1999; Hong et al., 2002; Smolyakov et al., 2003), or other unusual sites (McDonald \& Hourihane, 1972; Gargano et al., 2003). To our knowledge, there have been few reports of the parasite being located in the kidney, probably due to the distance between the kidney and the perianal area. Moreover, E. vermicularis granulomas are usually incidental and cause no clinical problems (Smolyakov et al., 2003), and in our case, the finding of the eggs of this parasite had no relation with the disease which led to the nephrectomy. The interrogation and the epidemiological investigation did not reveal when the patient had been infested by E. vermicularis.

\section{References}

Aydin, O. (2007). Incidental parasitic infestations in surgically removed appendices: a retrospective analysis. Diagn Pathol 2, 16.

Burkhart, C. N. \& Burkhart, C. G. (2005). Assessment of frequency, transmission, and genitourinary complications of enterobiasis (pinworms). Int J Dermatol 44, 837-840.

Cook, G. C. (1994). Enterobius vermicularis infection. Gut 35, 1159-1162.
Da Silva, D. F., da Silva, R. J., da Silva, M. G., Sartorelli, A. C. \& Rodrigues, M. A. (2007). Parasitic infection of the appendix as a cause of acute appendicitis. Parasitol Res 102, 99-102.

Dundas, K. C., Calder, A. A. \& Alyusuf, R. (1999). Enterobius vermicularis threadworm infestation of paraovarian tissue in a woman who has had a hysterectomy. Br J Obstet Gynaecol 106, 605607.

Gargano, R., Di Legami, R., Maresi, E. \& Restivo, S. (2003). Chronic sialoadenitis caused by E. vermicularis: case report. Acta Otorhinolaryngol Ital 23, 319-321.

Hong, S. T., Choi, M. H., Chai, J. Y., Kim, Y. T., Kim, M. K. \& Kim, K. R. (2002). A case of ovarian enterobiasis. Korean J Parasitol 40, 149151.

McDonald, G. S. A. \& Hourihane, D. O. B. (1972). Ectopic Enterobius vermicularis. Gut 13, 621-626.

Ramezani, M. A. \& Dehghani, M. R. (2007). Relationship between Enterobius vermicularis and the incidence of acute appendicitis. Southeast Asian J Trop Med Public Health 38, 20-23.

Smolyakov, R., Talalay, B., Yanai-Inbar, I., Pak, I. \& Alkan, M. (2003). Enterobius vermicularis infection of female genital tract: a report of three cases and review of the literature. Eur J Obstet Gynecol Reprod Biol 107, 220-222. 